ANNALES

POLONICI MATHEMATICI

$90.1(2007)$

\title{
Modularity of a nonrigid Calabi-Yau manifold with bad reduction at 13
}

by Grzegorz Kapustka and Michą Kapustka (Kraków)

\begin{abstract}
We identify the weight four newform of a modular Calabi-Yau manifold studied by Hulek and Verrill. The main obstacle is that this Calabi-Yau manifold is not rigid and has bad reduction at prime 13. Replacing the original fiber product of elliptic fibrations with a fiberwise Kummer construction we reduce the problem to studying the modularity of a rigid Calabi-Yau manifold with good reduction at primes $p \geq 5$.
\end{abstract}

1. Introduction. Consider the following elliptic fibrations:

$$
\begin{aligned}
& \mathcal{E}(\Gamma(3)):=\left\{\left(\left(t_{0}, t_{1}\right),(x, y, z)\right) \in \mathbb{P}^{1} \times \mathbb{P}^{2}:\left(x^{3}+y^{3}+z^{3}\right) t_{0}=3 x y z t_{1}\right\}, \\
& \mathcal{E}_{(1:-3:-3)}:=\left\{\left(\left(t_{0}, t_{1}\right),(x, y, z)\right) \in \mathbb{P}^{1} \times \mathbb{P}^{2}:\right. \\
&\left.(x+y+z)(x y-3(x z+y z)) t_{0}=x y z t_{1}\right\} .
\end{aligned}
$$

By the results of Schoen ([S]), the fiber product

$$
Y:=\mathcal{E}_{(1:-3:-3)} \times \mathcal{E}^{M t}(\Gamma(3)),
$$

where $\mathcal{E}^{M t}(\Gamma(3))$ is the twist of $\mathcal{E}(\Gamma(3))$ by the automorphism $M t=$ $(t+7) / 8$, has nodes as only singularities and its small resolution $\widehat{Y}$ is a (nonprojective) Calabi-Yau manifold with $h^{12}(\widehat{Y})=1$. Hulek and Verrill [HV] gave a method to prove the modularity of nonrigid Calabi-Yau manifolds based on the existence of many elliptic ruled surfaces. Using this method they proved the modularity of $\widehat{Y}$. More precisely, they showed that the $L$-series of $\widehat{Y}$ has the form

$$
L(\widehat{Y}, s)=L\left(g_{4}, s\right) L\left(g_{2}, s-1\right),
$$

where $g_{2}$ is the unique newform of weight 2 and level 13 , and $g_{4}$ is some newform of weight 4 . They also gave numerical evidence that $g_{4}$ is a newform of level 27 (27k4B in Stein's notation [St]). Let us point out that 13 is a

2000 Mathematics Subject Classification: 14G10, 14J32.

Key words and phrases: Calabi-Yau, modular forms, double coverings.

The project is co-financed from the European Union funds and national budget. 
prime of bad reduction for $\widehat{Y}$, but it does not divide the level of the weight 4 newform.

Our main goal is to prove that $g_{4}$ is the predicted newform. In fact, we shall consider an auxiliary Calabi-Yau manifold constructed by taking fiberwise the quotient by the natural involution. We can easily check that the resulting Kummer fibration is birational to the double cover $X$ of $\mathbb{P}^{3}$ (with coordinates $x, y, z, t$ ) branched along the octic surface

$$
-3 y(8 z+y-8 t)(x+t)\left(y^{2}-4 y z-8 t y-12 z^{2}\right)\left(x^{3}-3 t x^{2}+4 z^{3}\right)=0 .
$$

In Section 2, we shall prove that $X$ admits a nonprojective smooth model $\widehat{X}$, which is a rigid Calabi-Yau manifold. Using the Faltings-Serre-Livné method we prove in Section 3 that $\widehat{X}$ is modular with the newform $27 \mathrm{k} 4 \mathrm{~B}$. Since there is a generically $2: 1$ correspondence $\widehat{Y} \rightarrow \widehat{X}$, this proves the predicted form of the $L$-series of $\widehat{Y}$ (see Corollary 3.3). The advantage of replacing the fiber product by the Kummer fibration is that, after taking the quotient by the involution, 13 becomes a prime of good reduction, which drastically simplifies the computations.

Acknowledgements. We would like to express our gratitude to Sławomir Cynk for introducing us to the subject and for his enormous help in each aspect of the work on this paper.

2. Double octic. In this section, we shall study the smooth model of the double cover of $\mathbb{P}^{3}$ branched along the octic surface

$$
-3 y(8 z+y-8 t)(x+t)\left(y^{2}-4 y z-8 t y-12 z^{2}\right)\left(x^{3}-3 t x^{2}+4 z^{3}\right)=0 .
$$

Let us denote the singular double cover by $X$, and the components of the branch locus by $H_{1}, H_{2}, H_{3}, C_{2}, C_{3}$ respectively.

Proposition 2.1. The variety $X$ has a nonsingular (nonprojective) model that is a rigid Calabi-Yau manifold with Euler characteristic 48. Moreover $X$ has a partial resolution which is a nodal projective Calabi-Yau variety with 10 nodes.

Before the proof, let us study explicitly a suitable resolution of $X$. Let us first write down all singularities of $X$. They all correspond to singularities of the branch locus. On this branch locus there are 20 double curves, 5 fourfold points and 18 triple points. Let $\varepsilon$ and $\varepsilon^{2}$ be the two nontrivial complex third roots of unity.

Fourfold points:

$P_{1}=(1: 0: 0: 0)$ : The vertex of the quadric cone. It is a double point of $\mathrm{C}_{2}$ and lies on the hyperplanes $H_{1}$ and $H_{2}$. 
$P_{2}=(0: 1: 0: 0)$ : The vertex of the cubic cone. It is a triple point of $C_{3}$ and lies on $\mathrm{H}_{3}$.

$P_{3}=(-1: 0: 1: 1)$ : The intersection of $H_{1}, H_{2}, H_{3}$; it lies also on $C_{3}$.

$P_{4}=(0: 0: 0: 1)$ : The intersection of $H_{1}, C_{2}$ and $C_{3}$. It is a double point of $C_{3}$.

$P_{5}=(0: 8: 0: 1)$ : The intersection of $H_{2}, C_{2}$ and $C_{3}$. It is a double point of $C_{3}$.

Double lines:

$L_{1}, L_{2}, L_{3}$ : The lines $H_{1} \cap H_{2}, H_{1} \cap H_{3}, H_{2} \cap H_{3}$.

$L_{T}$ : The tangency line of $H_{1}$ and $C_{2}$.

$L_{21 a}, L_{21 b}$ : The two components of the intersection $H_{2} \cap C_{2}$.

$L_{32}$ : The conic which is the intersection $H_{3} \cap C_{2}$.

$L_{31 a}, L_{31 b}, L_{31 c}$ : The three lines that are components of the intersection $H_{3} \cap C_{3}$, where we denote by $L_{31 c}$ the line passing through $P_{3}$.

$K_{1}$ : The cubic $H_{1} \cap C_{3}$. It has a cusp at $P_{4}$.

$K_{2}$ : The cubic $H_{2} \cap C_{3}$. It has a cusp at $P_{5}$.

$S$ : The sextic $C_{2} \cap C_{3}$. It has two cusps at $P_{4}$ and $P_{5}$ and two nodes, which we denote by $N_{1}$ and $N_{2}$.

$D$ : The double line of the cubic cone $C_{3}$.

Triple points:

$H_{1} \cap H_{2} \cap C_{3}$ : One triple point, which we denote by $Q$. It is a tangency point of the line $L_{1}$ with $C_{3}$. It has coordinates $(2,0,1,1)$, hence is a rational point.

$H_{3} \cap C_{2} \cap C_{3}$ : Four triple points. Two of them are transversal triple points and two of them (denoted by $M_{1}, M_{2}$ ) are tangency points of the lines $L_{31 a}$ and $L_{31 b}$ with $C_{3}$. The tangency points have coordinates $M_{1}=(-1,4+2 \varepsilon, \varepsilon, 1)$ and $M_{2}=\left(-1,4+2 \varepsilon^{2}, \varepsilon^{2}, 1\right)$, hence they lie on the lines $P_{1} N_{1}$ and $P_{2} N_{2}$ respectively.

$H_{1} \cap C_{2} \cap C_{3}$ and $H_{1} \cap C_{2} \cap H_{3}$ : Two triple points, which we denote by $T_{1}$ and $T_{2}$, with coordinates $(-1,0,0,1)$ and $(3,0,0,1)$. They are not transversal, since $H_{1}$ is tangent to $C_{2}$.

$H_{1} \cap H_{3} \cap C_{3}$ : Two transversal triple points.

$H_{2} \cap H_{3} \cap C_{3}$ : Two transversal triple points.

$H_{2} \cap H_{3} \cap C_{2}$ : Two transversal triple points.

$H_{2} \cap C_{2} \cap C_{3}$ : Three transversal triple points.

To construct the resolution of $X$, we first blow up the fourfold points on the branch locus. Then we blow up all double curves that appeared on the proper transform of the branch locus and take the double cover of the resulting blow up of $\mathbb{P}^{3}$ branched over the proper transform of the branch locus. We denote the resulting variety by $\check{X}$. 
Let us look at the resolution of the singular points of the branch locus. We first blow up the fourfold points:

$P_{1}$ : After blowing up $P_{1}$, the proper transforms of the branch divisor intersect the exceptional divisor in two lines meeting in a point and a conic tangent to one of these lines at another point and intersecting the second line transversely. In particular the double curves of the proper transform of the branch divisor do not meet on the exceptional divisor.

$P_{2}$ : After blowing up $P_{2}$, the proper transforms of the branch divisor intersect the exceptional divisor in a line and a cuspidal conic meeting in three points. Here the double curves of the proper transform of the branch divisor also do not meet on the exceptional divisor.

$P_{3}$ : After blowing up $P_{3}$, the proper transforms of the branch divisor intersect the exceptional divisor in four lines no three meeting in a point. As before, the double curves of the proper transform of the branch locus are disjoint in a neighborhood of the exceptional divisor.

$P_{4}$ : After blowing up $P_{5}$, the proper transform of the cubic cone is tangent to the exceptional divisor along a line. The proper transforms of the remaining two components of the branch locus passing through $P_{5}$ meet each other transversely in another line on the exceptional divisor. Hence we obtain a new double curve on the branch locus, and we denote it by $B$.

$P_{5}$ : After blowing up $P_{4}$, the proper transform of the cubic cone is tangent to the exceptional divisor along a line. The proper transforms of the remaining two components of the branch locus passing through $P_{4}$ meet the exceptional divisor in two more lines.

After performing these blowings up, we obtain $\mathbb{P}^{3}$ blown up at five points and as the branch locus a surface without fourfold points. The branch locus is the union of $H_{1}$ blown up at three points, $H_{2}$ blown up at three points, $H_{3}$ blown up at two points, $C_{2}$ blown up at its vertex and two more points, and $C_{3}$ blown up at its vertex and three more points. Next we blow up all double curves on this new branch locus. Note that all of them are rational curves and all except $\widetilde{S}$, the transform of $S$, are smooth. There are 15 of them including $B$; however, we will need to blow up $L_{T}$ twice (this means that after blowing up $L_{T}$ the proper transforms of the cone $C_{2}$ and the hyperplane $H_{1}$ will still intersect in a line on the exceptional divisor). After these blowings up we obtain $\mathbb{P}^{3}$ blown up at five points and transformed by a sequence of 15 blowings up of smooth rational curves and the blow up of a rational curve with two nodes. Observe that this threefold has two nodes lying outside the branch locus; these are nodes that appeared after blowing 
up the nodal sextic $\tilde{S}$. The proper transform of the branch locus by this transformation is a surface without double curves and triple points. On the proper transform of $C_{3}$, when blowing up $B$, one node appears, while blowing up the proper transform of $D$ yields two nodes. After the first blowings up of lines passing through $Q, M_{1}$ and $M_{2}$, three more nodes appear. Hence $\check{X}$ is a nodal variety with ten nodes. We take the small resolution of the nodes and denote the resulting manifold (it is no more projective) by $\widehat{X}$.

Proof of Proposition 2.1. As in the above resolution of singularities we used only blowings up of fourfold points, double curves and a small resolution of nodes, the resolution $\widehat{X} \mapsto X$ is crepant. From the adjunction formula, we deduce that $\widehat{X}$ is a Calabi-Yau variety.

To compute the Euler characteristic we follow the above construction. The Euler characteristic of the transformed $\mathbb{P}^{3}$ is

$$
\chi\left(\widetilde{\mathbb{P}^{3}}\right)=4+5 \cdot 2+15 \cdot 2+1 \cdot 0=44 .
$$

To compute the Euler characteristic of the branch locus we need only observe that each blown up curve different from $B$ blows up one component of the branch locus at each triple point through which it passes. The number of blowings up at triple points is 19, moreover the exceptional divisor on $C_{3}$ after blowing up $B$ is also a line. This shows that the Euler characteristic of the branch locus is $15+(3+3+2+3+4)+19+1=50$. The Euler characteristic of $\check{X}$ is thus $2 * 44-50=38$. Taking a small resolution of the 10 nodes on the double cover $\check{X}$ we obtain a smooth variety $\widehat{X}$ with $\chi(\widehat{X})=48$.

The Hodge number $h^{12}$ of the Calabi-Yau manifold $\widehat{X}$ equals the dimension of the space of infinitesimal deformations. By [CvS] it is the dimension of the space of equisingular deformations of the branch locus in $\mathbb{P}^{3}$. We will prove that this space has dimension 0 , hence $X$ is rigid. Let us choose a coordinate system on $\mathbb{P}^{3}$ such that the points $P_{1}, P_{2}, P_{3}, P_{5}$ and $M_{1}$ correspond to $(1: 0: 0: 0),(0: 1: 0: 0),(0: 0: 1: 0),(0: 0: 0: 1),(1: 1: 1: 1)$.

The incidence and tangency conditions for the components of the branch locus yield a system of equations in the coefficients. Direct computations with SINGULAR ([GPS]) show that the system has a finite number of solutions.

3. Modularity. In this section, we shall prove the modularity of the Calabi-Yau manifold $\widehat{X}$. Since $\widehat{X}$ is not projective, the modulo $p$ reductions are not algebraic varieties. We can instead study the Galois action on the middle cohomology of the big resolution $\widetilde{X}$ of $X$ as this cohomology group can be naturally identified with $H^{3}(\widehat{X})$.

ThEOREM 3.1. The Calabi-Yau manifold $\widehat{X}$ is modular with cusp form of weight 4 and level 27. 
We will use the method of Faltings-Serre-Livné. First we compute the bad primes for our variety. Observe that the only candidates are 2, 3 and 13 .

Lemma 3.2. The reduction of $\widetilde{X}$ modulo 13 is smooth.

Proof. Reduction modulo 13 of the plane $\mathrm{H}_{2}$ passes through the points $M_{1}=(-1,-3,3,1)$ and $N_{1}=(2,-3,3,1)$. The branch locus around $M_{1}$ is

$$
(8 z+y-8 t)(x+t)\left(x^{3}-3 x^{2} t+4 z^{3}\right)\left(y^{2}-4 y z-8 y t-12 z^{2}\right)=0,
$$

hence it is a fourfold point.

Changing coordinates, the branch locus takes the form

$$
y x\left(y^{2}+6 y z+6 z^{2}+5 z\right)\left(x^{3}+4 z^{3}-6 x^{2}-3 z^{2}-4 x+4 z\right)=0 .
$$

After blowing up the line $\{x=0, y=0\}$ we have two affine charts. In one of them the branch locus is

$$
y\left(x^{2} y^{2}+6 x y z+6 z^{2}+5 z\right)\left(x^{3}+4 z^{3}-6 x^{2}-3 z^{2}-4 x+4 z\right)=0 .
$$

This is the equation of a transversal triple point at $(0,0,0)$, thus the singularity is resolved by further blowings up. In the second affine chart the branch locus is

$$
x\left(y^{2}+6 y z+6 z^{2}+5 z\right)\left(x^{3} y^{3}+4 z^{3}-6 x^{2} y^{2}-3 z^{2}-4 x y+4 z\right)=0 .
$$

Observe that the intersection

$$
\{x=0\} \cap\left\{x^{3} y^{3}+4 z^{3}-6 x^{2} y^{2}-3 z^{2}-4 x y+4 z=0\right\}
$$

splits into smooth and disjoint pieces. Only one of them needs to be considered more throughly. Blowing up the line $\{x=0, z=0\}$ we get one affine piece of the branch locus given by

$$
\left(y^{2}+6 x y z+6 x^{2} z^{2}+5 x z\right)\left(x^{2} y^{3}+4 x^{2} z^{3}-6 x y^{2}-3 x z^{2}-4 y+4 z\right)=0,
$$

the other affine piece contains only normal crossings. If we blow up the intersection of the above parts over $(0,0,0)$ we obtain a node on $\mathbb{P}^{3}$ incident with a node on the corresponding component of the branch locus. Taking a double cover we obtain a singularity that is not a node, but it is resolved by blowing up the point. Hence over the field $\mathbb{F}_{13}$ the singularity over $M_{1}$ is also resolved.

Over $N_{1}$ the situation is similar. After blowing up the line $M_{1} N_{1} \subset$ $H_{2} \cap C_{2}$ and the remaining singular intersection we obtain a node on $\mathbb{P}^{3}$ lying on the branch locus. We blow up the point and take the double cover obtaining a smooth surface.

Proof of Theorem 3.1. Since the reduction of $\widetilde{X}$ modulo $p$ is smooth for any prime $p \geq 5$, it remains to compute the number of points on the reductions $\widetilde{X}_{p}$ of $\widetilde{X}$ to $\mathbb{F}_{p}$ for primes $p \in\{5,7,11,13,17,19,23\}$. In these cases we can compute the number of points on $X_{p}$ using a $\mathrm{C}++$ program, then compute the number of points added during the resolution. For $p=$ 
13 the situation is different than in other cases. However, in the method of Faltings-Serre-Livné we can replace 13 by 37, hence from now on we assume $p \neq 13$. We will count separately the number of points added on the exceptional locus of each blowing up.

We first count the number of points added when blowing up the fourfold points. The method is to look at the number of points on proper transforms of lines lying on the exceptional divisors and passing through a chosen point. They are all double coverings of lines, hence have $1, p+1$ or $2 p+1$ points depending on the induced equations of the branch loci. Explicit computations yield

$$
\begin{aligned}
& \sharp E_{P_{1}}=\left\{\begin{array}{r}
(p-2)(p+1)+2(p+1)+2 p+1=p^{2}+2 p+1 \\
\text { if }-3 \text { is a square mod } p, \\
(p-2)(p+1)+2(p+1)+1=p^{2}+1 \\
\text { if }-3 \text { is not a square } \bmod p,
\end{array}\right. \\
& \sharp E_{P_{2}}=\left\{\begin{aligned}
&(p-2)(p+1)+3(2 p+1)-2 p=p^{2}+3 p+1 \\
& \text { if }-3 \text { is a square modulo } p, \\
& p(p+1)+(2 p+1)-2 p=p^{2}+p+1 \text { if }-3 \text { is not a square modulo } p,
\end{aligned}\right. \\
& \sharp E_{P_{3}}=p(p+1)+2 p+1-p=p^{2}+2 p+1, \\
& \sharp E_{P_{4}}=2 p^{2}+1 \text {, } \\
& \sharp E_{P_{5}}=2(p+1)+(2 p+1) \frac{p-1}{2}+\frac{p-1}{2}-p=p^{2}+p+1 \text {. }
\end{aligned}
$$

In addition to the above, we also need to count the points on the exceptional loci of the blowings up of all curves.

If we blow up a transversal double curve, we produce a conic bundle over some $\mathbb{P}^{1}$, where the fibers over triple points are lines. This gives $p^{2}+p$ new points for 13 transversal blowings up, together $13\left(p^{2}+p\right)$ new points.

If we blow up the tangency line, we get a double cover of $\mathbb{P}^{1} \times \mathbb{P}^{1}$ with coordinates $((a: b),(c: d))$ branched over the curve

$$
-3 c^{2}(a-b)(a-3 b)=0 \text {. }
$$

This adds $p^{2}+p$ points. The next blow up will be transversal.

On the blow up of $D$ we have $p^{2}+p$ new points, since the equation of the branch locus on the exceptional locus of the blow up is given by the product of two lines and the square of the section.

On the blow up of $S$ the number of added points is $p$ times the number of points on the curve $S$. We know that resolving the singularities on $S$ we get a smooth rational curve, hence consisting of $p+1$ points. However, by resolving a nodal curve over $\mathbb{F}_{p}$ we meet two possibilities. Either there appear 
two new points over the node or there are no points over it (i.e. we have lost one point by taking the resolution).

We have several possibilities:

- There were no nodes on $S$. This happens if -3 is not a square $\bmod p$. Then the number of points is the same as the number of points on the resolution, thus equals $p+1$.

- There were two nodes on $S$ and after blowing them up we get two points over each. This happens when both $\varepsilon-1$ and $\varepsilon^{2}-1$ are squares. Then the number of points on $S$ is $p-1$.

- There were two nodes on $S$ and after blowing them up we loose the two points. This happens when neither $\varepsilon-1$ nor $\varepsilon^{2}-1$ is a square. In this case the number of points on $S$ is $p+3$.

- There were two nodes, one of which vanished after the resolution and the other has been replaced by two points. This happens when exactly one of $\varepsilon-1, \varepsilon^{2}-1$ is a square. The number of points on $S$ in this case is $p+1$.

Taking all the above into consideration, we can give the number of points added before the small resolution of nodes:

$$
\sharp\left(\check{X}_{p}\right)-\sharp\left(X_{p}\right)= \begin{cases}22 p^{2}+24 p & \text { if }-3 \text { is a square mod } p \text { and exactly one of } \\ & \varepsilon-1, \varepsilon^{2}-1 \text { is a square, } \\ 22 p^{2}+22 p & \text { if }-3 \text { is a square mod } p \text { and both } \\ & \varepsilon-1, \varepsilon^{2}-1 \text { are squares, } \\ 22 p^{2}+26 p & \text { if }-3 \text { is a square mod } p \text { and none of } \\ & \varepsilon-1, \varepsilon^{2}-1 \text { is a square, } \\ 22 p^{2}+20 p & \text { if }-3 \text { is not a square } \bmod p .\end{cases}
$$

We next need to resolve the nodes that appeared on $\check{X}$. For this purpose, we perform a straightforward computation of the local equations of the nodes to decide how many points are added during the resolution.

We first check the complex nodes over the triple point $Q$. In this case we add

$$
\begin{cases}p^{2}+2 p & \text { if }-1 \text { is a square } \bmod p \\ p^{2} & \text { if }-1 \text { is not a square } \bmod p\end{cases}
$$

Three more nodes appear while blowing up $B$ and $D$. We can compute that resolving each of these nodes we always add $p^{2}+2 p$ new points.

Next, we check the nodes over $M_{1}$ and $M_{2}$. The points $M_{1}$ and $M_{2}$ have rational coordinates depending on the rationality of $\varepsilon$. The latter is rational if and only if -3 is a square in $\mathbb{F}_{p}$. 
Over $\mathbb{F}_{p}$ for which -3 is a square, resolving the nodes $M_{1}$ and $M_{2}$ adds $\begin{cases}2\left(p^{2}+2 p\right) & \text { if } 3-\varepsilon, 3-\varepsilon^{2} \text { are squares } \bmod p, \\ p^{2}+2 p+p^{2} & \text { if exactly one of } 3-\varepsilon, 3-\varepsilon^{2} \text { is a square mod } p \\ 2 p^{2} & \text { if none of } 3-\varepsilon, 3-\varepsilon^{2} \text { is a square } \bmod p .\end{cases}$

We also have four complex nodes that appeared outside the branch locus when blowing up the curve $S$. For this case there are three possibilities:

- There are no rational nodes; this happens if -3 is not a square or the value of the polynomial defining the branch locus is not a square at these points. That occurs when none of $5 \varepsilon-2,5 \varepsilon^{2}-2$ is a square $\bmod p$.

- There are two rational nodes; this happens if the value of the polynomial defining the branch locus at one of the nodes is a square. That occurs when exactly one of $5 \varepsilon-2,5 \varepsilon^{2}-2$ is a square $\bmod p$.

- There are four rational nodes; this happens if the value of the polynomial defining the branch locus at both nodes is a square. That occurs when both $5 \varepsilon-2,5 \varepsilon^{2}-2$ are squares $\bmod p$.

Moreover, for each of these cases the resolution of the nodes adds either $p^{2}+2 p$ or $p^{2}$ new points. The actual case is determined by the answer to the question: "Is $1-\varepsilon$ a square $\bmod p$ ?".

Hence the number of points added by taking the big resolution $\tilde{X}$ of $X$ depends on $p$ in the following way.

Let

$$
\begin{aligned}
f(p)= & \left(\frac{3-\varepsilon}{p}\right)+\left(\frac{3-\varepsilon^{2}}{p}\right)+\left(1+\left(\frac{5 \varepsilon-2}{p}\right)\right) \cdot\left(\frac{1-\varepsilon}{p}\right) \\
& +\left(1+\left(\frac{5 \varepsilon^{2}-2}{p}\right)\right) \cdot\left(\frac{1-\varepsilon^{2}}{p}\right)-\left(\frac{\varepsilon-1}{p}\right)-\left(\frac{\varepsilon^{2}-1}{p}\right),
\end{aligned}
$$

where $\left(\frac{a}{b}\right)$ is the Legendre symbol. Then

$$
\sharp\left(\widetilde{X}_{p}\right)-\sharp\left(X_{p}\right)= \begin{cases}26 p^{2}+28 p & \text { if }-3 \text { is not a square mod } p, \\ 28 p^{2}+34 p+f(p) p & \text { if }-3 \text { is a square mod } p \text { and none } \\ 30 p^{2}+36 p+f(p) p & \text { of } 5 \varepsilon-2,5 \varepsilon^{2}-2 \text { is a square mod } p, 3 \text { is a square mod } p \text { and one } \\ & \text { of } 5 \varepsilon-2,5 \varepsilon^{2}-2 \text { is a square mod } p, \\ 32 p^{2}+38 p+f(p) p & \text { if }-3 \text { is a square mod } p \text { and both } \\ & 5 \varepsilon-2,5 \varepsilon^{2}-2 \text { are squares mod } p .\end{cases}
$$

The following table presents the explicit numbers needed in the method of Faltings-Serre-Livné: 


\begin{tabular}{lccr}
\hline$p$ & $\sharp\left(X_{p}\right)$ & $\sharp\left(\widetilde{X}_{p}\right)$ & $a_{p}$ \\
\hline 5 & 191 & 981 & -15 \\
7 & 439 & 2273 & -25 \\
11 & 1559 & 5013 & 15 \\
37 & 53674 & 96076 & -430 \\
17 & 5564 & 13554 & -72 \\
19 & 7504 & 18258 & 2 \\
23 & 13340 & 27738 & -114 \\
\hline
\end{tabular}

Comparing the values of $\sharp\left(\widetilde{X}_{p}\right)$ with the coefficients $a_{p}$ of the cusp form from [St] we conclude the proof of the modularity of $\widehat{X}$.

Using the 2:1 correspondence $\widehat{Y} \rightarrow \widehat{X}$ we obtain the following.

COROLlary 3.3. The variety $\widehat{Y}$ is a modular Calabi-Yau manifold with the L-series $L(\widehat{Y}, s)=L\left(g_{4}, s\right) L\left(g_{2}, s-1\right)$, where $g_{2}$ is the unique newform of weight 2 and level 13 , and $g_{4}$ is the newform $27 \mathrm{k} 4 \mathrm{~B}$ of weight 4 and degree 27.

\section{References}

[CvS] S. Cynk and D. van Straten, Infinitesimal deformations of smooth algebraic varieties, Math. Nachr. 279 (2006), 716-726.

[GPS] G.-M. Greuel, G. Pfister and H. Schönemann, Singular 2.0. A Computer Algebra System for Polynomial Computations, Centre for Computer Algebra, University of Kaiserslautern, 2001; http://www.singular.uni-kl.de.

[HKS] K. Hulek, R. Kloosterman and M. Schütt, Modularity of Calabi-Yau varieties, preprint, math.AG/0601238.

[HV] K. Hulek and H. Verrill, On the modularity of Calabi-Yau threefolds containing elliptic ruled surfaces, preprint, 2005, math.AG/0502158, to appear in AMS/IP Studies in Advanced Mathematics "Mirror Symmetry V", Proceedings of the BIRS Workshop on Calabi-Yau varieties and mirror symmetry, December 6-11, 2003.

[M] Ch. Meyer, Modular Calabi-Yau Threefolds, Fields Inst. Monogr. 22, Amer. Math. Soc., 2005.

[S] C. Schoen, On fiber products of rational elliptic surfaces with section, Math. Z. 197 (1988), 177-199.

[St] W. A. Stein, Modular forms database, http://modular.math.washington.edu/.

Institute of Mathematics

Jagiellonian University

Reymonta 4

30-059 Kraków, Poland

E-mail: Grzegorz.Kapustka@im.uj.edu.pl

Michal.Kapustka@im.uj.edu.pl

Received 14.9.2006

and in final form 3.11.2006 